Pacific Journal of Mathematic 


\title{
DISTRIBUTIVE LATTICES WITH FINITE PROJECTIVE COVES
}

\author{
Alan Day, Herb Gaskill, and Werner Poguntke
}

A projective cover in a category, $\mathscr{K}$, of algebras is a morphism $\phi: P \rightarrow A$ where $A, P \in \mathscr{K}, P$ is projective in $\mathscr{K}$ and no proper subalgebra of $P$ has $A$ as its direct image under $\phi$. Projective covers of finite distributive lattices in the categories $\mathscr{L}$, of all lattices and $\mathscr{L}_{\text {fin }}$ of all finite lattices are determined (when they exist).

1. Introduction. The notion of a projective algebra in a given variety of algebras, $\mathscr{K}$, is well-known and coincides with the categorical notion of $\mathscr{E}$-projectivity in $\mathscr{K}$, qua category, where $\mathscr{E}$ is the class of all surjective homomorphisms in $\mathscr{K}$ (c.f. Banaschewski [5]). Moreover, since such $\mathscr{K}$-projective algebras (the $\mathscr{E}$ will be fixed as above throughout) will be retracts of $\mathscr{K}$-free algebras, the study of $\mathscr{K}$-projectives is ultimately linked with the study of subalgebras of $\mathscr{K}$-free algebras.

In the case of $\mathscr{L}$, the variety of all lattices, the sublattice problem was the first to receive consideration (cf. [11], [13], [18], [19] and especially Jónsson and Nation [20]). The first serious treatment of projective lattices seems to have been McKenzie's monumental paper, [22], where he showed (among other things) that every finite sublattice of a free lattice is projective. Kostinsky [21], extended this result to finitely generated lattices and Freese and Nation, [11] completely characterized arbitrary projective lattices.

Returing to finite lattices, several characterizations of finite projective lattices have been found (cf. [22], [16], [17]). Most of these have been shown interrelated ([11], [17], [20]) and all have fallen short of proving Jónsson's conjecture that finite sublattices of free lattices are characterized by the two semidistributive implications, $\left(S D_{\wedge}\right)$ and $\left(S D_{\vee}\right)$ together with Whitman's condition, $(W)$.

In attempting to prove Jónsson's conjecture and in constructing examples of finite projective lattices conflicts between $(W)$ and finiteness often appear (cf. [20; Lemma 7.4]). In an attempt to clarify the connection the authors and several others ${ }^{1}$ in the area have (at present) unpublished examples of finite posets and partial lattices which if embedded in a lattice satisfying $(W)$ force this lattice to be infinite. This relation between $(W)$ and infiniteness is still not completely understood.

1 B. Sands and I. Rival, Planar Sublattices of a Free Lattice, Canad. J. Math., 30 (1978), 1256-1283. 
The purpose of this paper is to consider a method of producing finitely generated projective lattices from finite distributive lattices and to provide necessary and sufficient conditions (on the finite distributive lattice) for the so-produced projective lattice to be finite. Hopefully this will provide a beginning in analyzing the $(W)$-infiniteness connection. We also present three structure theorems. The first classifies the "good" finite distributive lattices by exclusion of certain sublattices; the second characterizes the resultant finite projective lattices internally, and the third describes the "building blocks" from which one produces these finite projective lattices.

The main ideas and results in this paper have had a long gestation period. They were first obtained (in a very different form) by the second author and circulated by him in preprint form (Gaskill [14] and [15]). The other authors determined that his results fit nicely into the theory of projective covers and that in this theory, Gaskill's main theorems could by simplified into their present form.

2. Preliminaries. Let $\mathscr{K}$ be a class of lattices. A lattice $P \in \mathscr{K}$ is called $\mathscr{K}$-projective if for any $A, B \in \mathscr{K}$, epimorphism (=surjection) $\phi: A \rightarrow B$ and map $\psi: P \rightarrow B$, there exists a "lift" $\bar{\psi}: P \rightarrow A$ with $\dot{\phi} \circ \bar{\psi}=\psi$. An epimorphism $\phi: A \rightarrow B$ is called $\mathscr{K}-$ essential ("tight" is another name in the literature) if for any other $C \in \mathscr{\mathscr { K }}$ and $\psi: C \rightarrow A, \phi \circ \psi$ is surjective if and only if $\psi$ is. All of the classes of lattices we will consider here are closed under the formation of sublattices and in this case $\dot{\phi}: A \rightarrow B$ is essential if and only if no proper sublattice of $A$ has $B$ as its image under $\phi$. A $\mathscr{K}$-projective cover is an essential epimorphism $\phi: P \rightarrow B$ where $P$ is $\mathscr{K}$-projective. These notions are completely categorical in nature and we suggest Banaschewski [5] as a general reference.

The classes of lattices we are interested in are $\mathscr{L}$, the variety of all lattices, $\mathscr{L}_{\text {in }}$, the class of all finite lattices, and $\mathscr{D}$, the variety of all distributive lattices. The projective lattices in each of these classes have been described in Freese and Nation [12], Davey and Sands [7], and Balbes and Horn [3], respectively. In [4] projective covers in $\mathscr{D}$ are described. Since we will be dealing only with finitely generated lattices, we give the characterizations of them.

THeOREM 2.1 ([7] and also [22]). $L \in \mathscr{L}_{\text {fin }}$ is projective if and only if it satisfies Whitman's condition $(W): a \wedge b \leqq c \vee d$ implies $\{a, b, c, d\} \cap[a \wedge b, c \vee d] \neq \phi$.

THEOREM 2.2 ([2]). A finite (=finitely generated) distributive

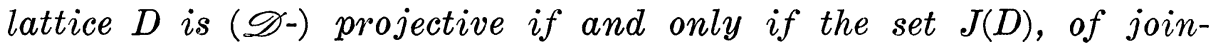
irreducibles is closed under the meet operation. 
THEOREM 2.3 ([21]; cf. also [22], and [19]). For a finitely generated $L \in \mathscr{L}$, the following are equivalent:

(1) $L$ is projective

(2) $L$ is a sublattice of a free lattice

(3) L satisfies $(W)$ and belongs to $\mathscr{B}$, where $\mathscr{B}$ is the class of all finitely generated lattices, L, satisfying: $A$, a finitely generated lattice, $f: A \rightarrow L$ an epimorphism then for all $x \in L, f^{-1}(x)=[\alpha(x), \beta(x)]$ for some bounded injections $\alpha, \beta: L \succ A$.

We also need the "splitting of intervals" construction given originally in Day [8]. If $A$ is a lattice and $I=[u, v]$ is an interval of $A$, then $A[I]=(A \backslash I) \cup(I \times 2)$ and the original (and first projection) order relating $A \backslash I$ and $I \times 2$. There is a canonical epimorphism $\kappa: A[I] \rightarrow A$.

LEMMA 2.4 ([9]). If $A \in \mathscr{B}$ then $A[I] \in \mathscr{B}$ for all intervals $I \subseteq A$.

An interval $[u, v] \subseteq A$ is called a $(W)$-failure interval if there exists $a, b, c, d, \notin[u, v]$ such that $a \wedge b=u$ and $c \vee d=v$.

Lemma 2.5. For a lattice $A$ and interval $I \leqq A, \kappa: A[I] \rightarrow A$ is essential if and only if $I$ is a (W)-failure interval.

3. Projective covers in $\mathscr{L}$ and $\mathscr{L}_{\text {in }}$ Even though in $\mathscr{L}$, every lattice is a homomorphic image of a projective lattice (free lattices are projective), it does not follow that every lattice has a projective cover in $\mathscr{L}$. An example of such a lattice is $M_{3}$ in Figure (i). Suppose $P=\langle x, y, z\rangle \stackrel{f}{\rightarrow} M_{3}$ is a projective cover. If $x \not y \vee z$ then $x$ is join-prime in $P$. Also since $f$ is essential, $P$ would be generated by $\{x \wedge(y \vee z), y, z\}$. But then $x$ would have to be a meet of these generators which is impossible. Hence we have in $P, x \vee y=x \vee z=y \vee z$. But by $\left(S D_{\vee}\right)$ we get $x \vee y=x \vee z=$ $x \vee(y \wedge z)$. Therefore by applying $f, 1=a$, a contradiction.

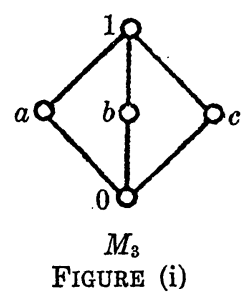

The following result gives the existence of at least some lattices with projective covers in $\mathscr{L}$.

TheOREM 3.1. Every finite distributive lattice, D, has an $\mathscr{L}-$ projective cover. 
Proof. For a suitable finite set, $X$, consider the bounded epimorphism $f: F L(X) \rightarrow D$ with lower and upper bounds $\alpha, \beta: D \succ F L(X)$, respectively. Now $P=\langle\beta[M(D)]\rangle \leqq F L(X)$ is finitely generated and therefore $g=f \uparrow P$ is bounded with lower and upper bounds $\alpha_{P}, \beta_{P}: D \succ$ $P$, respectively. Moreover $\beta_{P}=\beta$. We compute $\alpha_{P}$ at a join-prime $u \in J(D)$.

$$
\begin{aligned}
\alpha_{P}(u) & =\wedge\{q \in \beta[M(D)]: u \leqq g(q)\} \\
& =\wedge\{\beta(m): m \in M(D) \text { and } u \leqq m\} \\
& =\beta(u) .
\end{aligned}
$$

Now let $Q=\left\langle\alpha_{P}[J(D)]\right\rangle$ and $h=f\lceil Q . \quad h$ is surjective, and $Q$ is projective by 2.3. Moreover as $h^{-1}(u)$ is a singleton for all $u \in J(D), h$ is essential. Therefore $h: Q \rightarrow D$ is an $\mathscr{L}$-projective cover.

For the category, $\mathscr{L}_{\text {in }}$, one can easily see that having an $\mathscr{L}_{\text {in }}$ projective cover is equivalent with being the homomorphic image of an $\mathscr{L}_{\text {in }}$-projective. For the case of finite distributive lattices, the relationship between the two possible projective covers is explicit.

THEOREM 3.2. A finite distributive lattice possesses an $\mathscr{L}_{\mathrm{fin}^{-}}$ projective cover if and only if its $\mathscr{L}$-projective cover is finite. In that case, the two projective covers are equal.

Proof. Since projective lattices satisfy $(W)$, the condition is sufficient for the existence of an $\mathscr{L}_{\text {in }}$-projective cover.

Conversely let $P \stackrel{f}{\rightarrow} D$ be the $\mathscr{L}_{\text {in }}$-projective cover of $D$. We must show that $P$ is projective in $\mathscr{L}$. But $D \in \mathscr{B}$ and as $P$ is finite we can reach $P$ in a finite number of steps by splitting $(W)$-failure intervals. Since $\mathscr{B}$ is closed under the splitting of intervals, we obtain $P \in \mathscr{B}$ and therefore by $2.3 P$ is projective in $\mathscr{L}$.

It is of interest then to know which finite distributive lattices have finite projective covers in $\mathscr{L}$. If $\mathscr{C}$ is the class of all such distributive lattices, then it is clear that $\mathscr{C}$ is closed under the formation of subalgebras and homomorphic images. Our next result shows that $\mathscr{C}$ is not all distributive lattices. (Figure (ii))
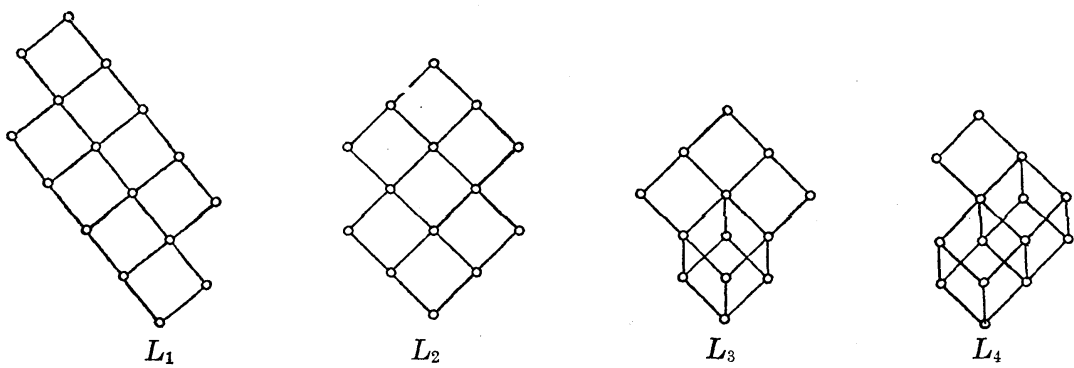

Figure (ii) 
THEOREM 3.3. $L_{1}, L_{2}, L_{3}, L_{4}, L_{3}^{d}$ and $L_{4}^{d}$ are not members of $\mathscr{C}$.

Proof. We consider $L_{3}$ first. Easy checking shows that the map $f$ in Figure (iii) is an essential epimorphism. Noting that another copy of $L_{3}$ appears in the domain of $f$, we see that an iterated sequence of essential epimorphisms can be constructed, with no bound on their cardinality. Since the projective cover of $L$ must factor through each of these it cannot be finite.
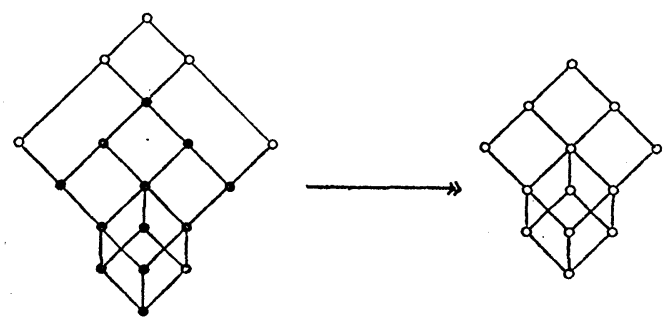

FIgURE (iii)

Re $L_{2}$ : The map in Figure (iv) is essential and its domain has $L_{3}$ as a sublattice.

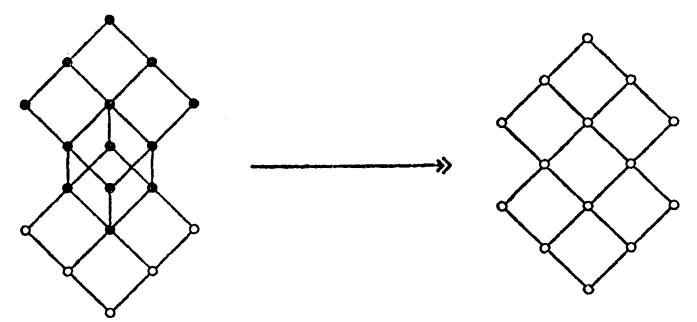

Figure (iv)

Re $L_{1}$ : The map in Figure (v) is essential and its domain has $L_{1}$ as a sublattice.

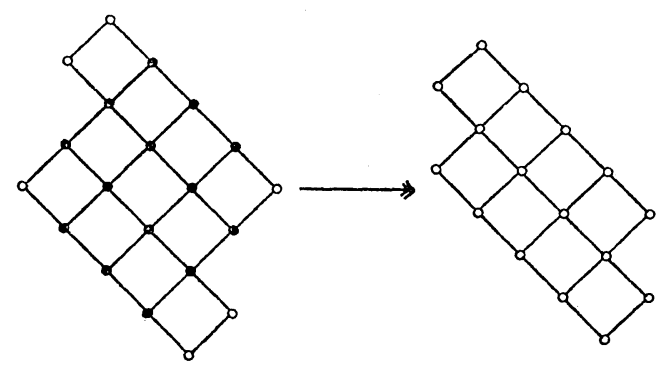

Figure (v)

Re $L_{4}$ : The map in Figure (vi) is essential and its domain has $L_{3}$ as a sublattice. 

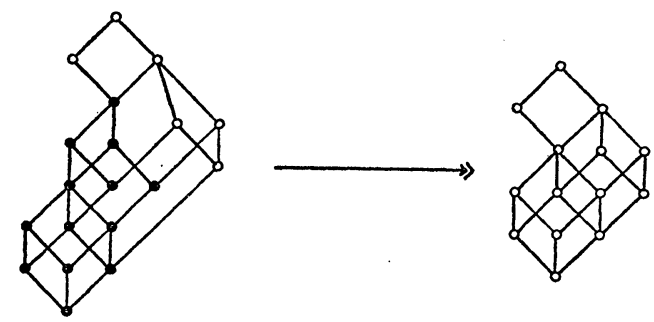

Figure (vi)

It is clear that a lattice belongs to $\mathscr{C}$ if and only if its dual does.

Now $\mathscr{C}$ is closed under the formation of subalgebras so therefore is definable by the exclusion of certain sublattices. The remainder of this paper will be devoted to showing that the six mentioned in the above list provide such a definable list. That is: - if $\mathscr{C}=$ $\left\{D \in \mathscr{D}_{\mathrm{fin}}: \forall i=1,2,3,4, L_{i} \not \equiv D\right.$ and $\left.L_{i}^{d} \not \equiv D\right\}$ then $\mathscr{G}=\mathscr{C}$.

4. The class $\mathscr{G}$ and the first reduction. As has been mentioned earlier, a finite projective cover of a finite distributive lattice will be produced after a finite number of splittings of $(W)$-failure intervals. Two points then become of great interest.

Firstly, by adroit selection, we can initially split the $(W)$-failure intervals that keep the resultant lattice distributive. Such an interval is called a distributive (W)-failure interval. It is clear that $[u, v] \subseteq L$ is such iff $L=[u, \rightarrow) \cup(\leftarrow, v]$. This produces the $\mathscr{D}$-projective cover $P_{c}(D) \stackrel{f}{\rightarrow} D$ of the original lattice $D$.

Secondly, it will be important to know the structure of $(W)$ failure intervals of members of $\mathscr{G}$. We answer the second question first.

THEOREM 4.1. For $D \in \mathscr{G}$, let $[u, v]$ be a proper $(W)$-failure interval in $D$. Then there exists $p, q, r, s \in D$ that generate a sublattice of $D$ isomorphic to $N_{i}$ in Figure (vii).
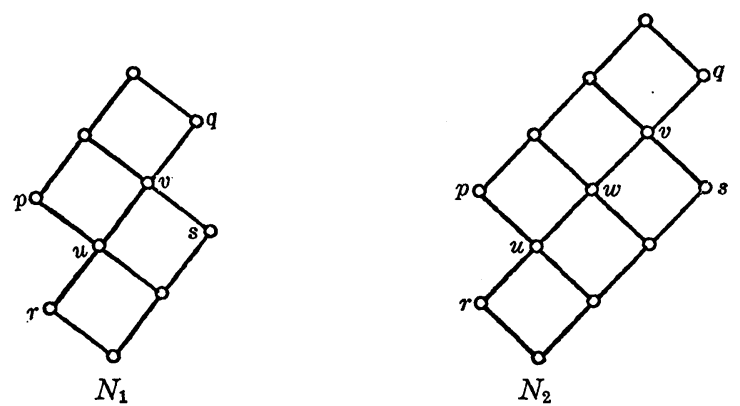

Figure (vii) 
Proof. By assumption there exists $a, b, c, d \in D$ with $a \wedge b=u$, $c \vee d=v$ and $\{a, b, c, d\} \cap[u, v]=\phi$.

Now consider the sublattice of $D$ generated by $\{a, b, c \vee d\}$ which must be a homomorphic image of the lattice in Figure (viii) with $x=$ $a, y=c \vee d$ and $z=b$.

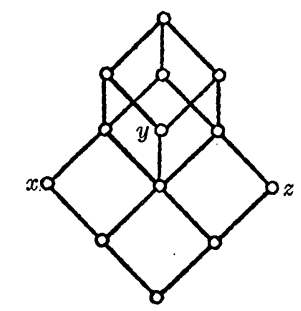

$F D(x, y, z: x \wedge z \leqq y)$

FIgURE (viii)

Since the lattice itself is a forbidden sublattice, the homomorphic image must be a proper one and therefore one of the following must hold
(A) $a \wedge(c \vee d) \leqq b$
(B) $b \wedge(c \vee d) \leqq a$
(C) $c \vee d \leqq a \vee b$.

Dually by considering the sublattice of $D$ generated by $\{a \wedge b, c, d\}$ we must have one of:

$\left(\mathrm{A}^{\prime}\right) \quad c \leqq d \vee(a \wedge b)$

$\left(\mathrm{B}^{\prime}\right) \quad d \leqq c \vee(a \wedge b)$

$\left(\mathrm{C}^{\prime}\right) \quad c \wedge d \leqq a \wedge b$.

Now if $(A)$ and $\left(A^{\prime}\right)$ hold, we have

$$
a \wedge(b \vee c \vee d)=a \wedge b=u
$$

and

$$
d \vee(a \wedge b \wedge c)=d \vee c=v
$$

Therefore, let $p=a, q=b \vee c \vee d, r=a \wedge b \wedge c$, and $s=d$.

If $(\mathrm{A})$ and $\left(\mathrm{C}^{\prime}\right)$ hold, then without loss of generality $b=b \vee c \vee d$. But $\left(\mathrm{C}^{\prime}\right)$ forces the sublattice generated by $\{a, c, d\}$ to also be a homomorphic image of the lattice in Figure (viii) with $x=c, y=a$, and $z=d$.

Since $a \not \leq \vee d, a \wedge b \not c$, and $a \wedge b \not d$ follow from our $(W)$ failure assumption, we must have

$$
c \leqq a \wedge b \text { or } d \leqq a \wedge b
$$

either of which give us our desired sublattice of $D$. 
Now by symmetry and duality, we need only check the possibility of $(\mathrm{C})$ and $\left(\mathrm{C}^{\prime}\right)$ holding while the four other conditions do not. This produces the two overlapping sublattices of $D$ in Figure (ix).
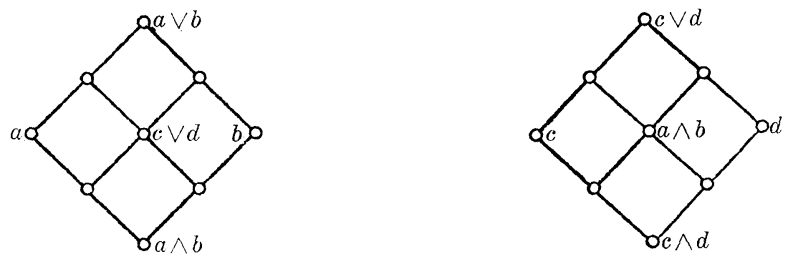

Figure (ix)

Now $a \wedge b<c \vee(a \wedge b)<c \vee d$ forces it to be comparable with $a \wedge(c \vee d)$ or $b \wedge(c \vee d)$ since $D$ does not contain $L_{3}$. But then as both pairs $\{a \wedge(c \vee d), b \wedge(c \vee d)\}$ and $\{c \vee(a \wedge b), d \vee(a \wedge b)\}$ are complemented pairs, we must have $\{a \wedge(c \vee d), b \wedge(c \vee d)\}=\{c \vee(a \wedge b)$, $d \vee(a \wedge b)\}$. But this implies $L_{2} \leqq D$, a contradiction. Therefore this case cannot occur.

COROLlaRY 4.2. 1. If $D$ does not contain any of the forbidden sublattices and $[u, v]$ is a proper $(W)$-failure interval in $D$, then $|[u, v]| \leqq 3$.

Proof. Consider the sublattice of $D$ given by the theorem. If $[u, v]$ contained a four-element chain, we would have $L_{1} \leqq D$. Therefore, all chains in $[u, v]$ have less than four members.

Now, if there are two incomparable members of $[u, v]$, say, $x$ and $y$, the condition on chains forces $x \vee y=v$ or $x \wedge y=u$. However, the first case implies $L_{4} \leqq D$ and the second, $L_{4}^{d} \leqq D$.

COROLlary 4.3. 2. If $D$ does not contain any of the forbidden sublattices and $[u, v]$ is a proper $(W)$-failure interval in $D$, then there are precisely two possibilities for the sublattices guaranteed by the theorem; namely, $N_{1}$ and $N_{2}$ (Figure (vii)). Moreover,

(1) All covering relations in the sublattice are covering relations in $D$;

(2) $u$ (and $w$ ) have precisely two covers in $D$;

(3) $v$ (and $w$ ) cover precisely two elements of $D$.

LEMMA 4.4. $\mathscr{G}$ is closed under homomorphic images.

Proof. Consider an epimorphism $f: A \rightarrow B$. If $B$ contains one of $L_{3}, L_{4}$ and their duals, then so does $A$ as these are projective. Suppose then that $L_{1} \leqq B$ and consider $g: P_{\mathscr{S}}\left(L_{1}\right) \rightarrow L_{1}$, which is given 
in Figure (iv). Since $P_{\mathscr{D}}\left(L_{1}\right)$ is projective, there exists $\bar{g}: P_{\mathscr{D}}\left(L_{1}\right) \rightarrow A$ with $f \circ \bar{g}=g$. Since $\operatorname{Ker} \bar{g} \leqq \operatorname{Ker} g$, either $\operatorname{Ker} \bar{g}=\Delta_{P_{\mathscr{g}\left(L_{1}\right)}}$ or $\operatorname{Ker} \bar{g}=$ Ker $g$. Therefore, $L_{2} \leqq A$ or $L_{1} \leqq A$, respectively. The case for $L_{2} \leqq B$ is similar as Figure (iii) gives us $P_{\mathscr{D}}\left(L_{2}\right)$.

LEMMA 4.5. $\mathscr{G}$ is closed under the splitting of distributive $(W)-$ failure intervals.

Proof. Take $D \in \mathscr{G}$ and let $I=[u, v]$ be a $(W)$-failure interval with $D=[0, v] \cup[u, 1]$. Assume further that for some $i=1,2,3,4$, $L_{i} \leqq D[I]$. (The dual possibilities will follow dually.) Since $D \in \mathscr{G}$, $\operatorname{Ker}\left(\kappa \uparrow L_{i}\right)$ is a proper congruence on $L_{i}$ and since $|[u, v]| \leqq 3$, it has at most three nontrivial congruence classes. We must then look at all such possible congruences on the $L_{i}$ and, in order to work in $D$, the possible $\kappa\left[L_{i}\right]$. The contradictions will come from attempts to fit together these $\kappa\left[L_{i}\right]$ with the $(W)$-failure sublattice of $D$ given by the last theorem. We prove these two cases and leave the rest for the reader.

Case (i). $i=3$ and $\kappa\left[L_{3}\right]$ is given by Figure (x). This forces the ( $W$ )-failure sublattice of $D$ to be $N_{2}$. But this contradicts Corollary 2 on the number of elements $w$ can cover.

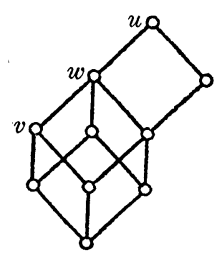

Figure (x)

Case (ii). $i=1$ and $\kappa\left[L_{1}\right]$ is given by Figure (xi). But $q>v$ implies $L_{2} \leqq D$, a contradiction.

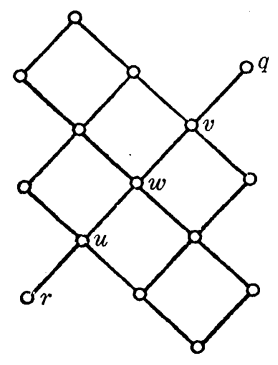

FIgURE (xi)

THEOREM 4.6. $D \in \mathscr{G}$ if and only if its $\mathscr{D}$-projective cover $P_{\mathscr{S}}(D) \in \mathscr{G}$. 
Proof. If $P_{\mathscr{Z}}(D) \in \mathscr{G}$, then so is $D$ by 4.4. Conversely, if $D \in \mathscr{G}$, then so is $P_{\mathscr{T}}(D)$ as $P_{\mathscr{D}}(D)$ can be obtained from $D$ by a sequence of maps $D_{i+1}=D_{i}\left[I_{i}\right] \rightarrow D_{i}$ where $I_{i}$ is a distributive $(W)$-failure interval in $D_{i}$. Therefore 4.5 applies.

The above results allow us then to consider only $\mathscr{D}$-projective distributive lattices in our proof of the converse $G \subseteq \mathscr{C}$. The next section will give the final reduction and this proof.

5. Final reduction and the main theorem. In this section, we first introduce the notion of a $\square$-decomposition of a finite lattice. The characterization of the projective $\square$-indecomposables in $\mathscr{G}$ will then help us to prove the main theorem.

Definition 5.1. Let $K$ be a finite lattice. A sequence $\left(K_{1}, \cdots, K_{n}\right)$ of intervals $K_{i}$ of $K$ is called a $\square$-decomposition of $K$ if and only if the following holds:

(i) $\bigcup_{1 \leqq i \leqq n} K_{i}=K$;

(ii) for every $1 \leqq i \leqq n-1$, there is a coatom $a_{i}$ in $K_{i}$ and an atom $b_{i+1}$ in $K_{i+1}$ such that $a_{i} \prec 0_{K_{i+1}}, 1_{K_{i}} \prec b_{i+1}$, and $\left[0_{K_{i}}, 1_{K_{i+1}}\right]=$ $K_{i} \cup K_{i+1}$. See Figure (xii).

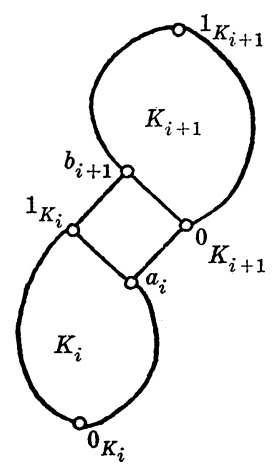

FIgURE (xii)

$K$ is $\square$-indecomposable if for any such $\square$-decomposition it follows that $n=1$.

The following lemma is easily proved by induction:

Lemma 5.2. Let $K$ be a finite lattice. Then $K$ can be uniquely $\square$-decomposed into $\square$-indecomposable sublattices.

We next characterize the projective $\square$-indecomposables in $\mathscr{G}$.

THEOREM 5.3. Let $D$ be a finite projective distributive lattice which is linearly and $\square$-indecomposable. If $D \in \mathscr{G}$, then $D$ is isomorphic to $2^{4}$ or to a sublattice of $2^{2} \times 5$. 
Proof. Let us first recall that a distributive lattice $L$ has breadth $n(b(L)=n)$ if and only if it has a sublattice isomorphic to $2^{n}$, but none which is isomorphic to $2^{n+1}$. Equivalently, the width of the partially orderd set $J(L)$ equals $n$. This also implies that no element of $L$ has more than $n$ upper (or lower) covers.

Since $L_{3}$ as well as $L_{4}$ are sublattice of $2^{5}, b(D) \leqq 4$. But as $D$ is linearly indecomposable, it is easy to see that $b(D)=4$ already implies $D \cong 2^{4}$. So the proof of 5.3 will be finished if we prove the following two claims:

Claim 1. If $b(D)=3$, then $D \cong 2^{2} \times k$ for some $2 \leqq k \leqq 5$.

Claim 2. If $b(D)=2$, then $D \cong 3 \times k$ for some $3 \leqq k \leqq 5$.

Proof of Claim 1. Let $C$ be a sublattice of $D$ isomorphic to $2^{3}$ such that covers in $C$ are covers in $D$; let $a, b, c$ be the atoms of $C, e:=a \wedge b$. We first notice that if $a$ and $b$ are join-reducible, then in order not to generate $L_{3}^{d}$ as a sublattice of $D$ the lower covers $e \neq u-<a$ and $e \neq v-<b$ must satisfy $u \wedge v=u \wedge e=v \wedge e$ which forces a cover-preserving sublattice of $D$ isomorphic to $2^{2} \times 3$. It also follows that in this case, $c, e \in J(D)$ since $L_{4}^{d} \not \leq D$.

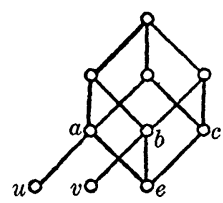

Figure (xiii)

Suppose now that $b$ and $c$ are join-irreducible. We will show that $a$ is also in $J(D)$ and $e=0$.

Assume to the contrary that $a$ has another lower cover $u \neq e$. Since $D$ is projective in $\mathscr{D}, e=b \wedge c \in J(D)$ and $e \wedge u$ must be the unique lower cover of $e$ (see Figure (xiv)). Since $D$ is $\square$-indecomposable, $D \neq[0, u] \cup[e, 1]$ and therefore $u<f=\bigvee\{x \in D: x \wedge e=e \wedge u\}$. Moreover $b \not f$ and $c \not f$ imply there exists an upper cover, $v$, of a distinct from $a \vee b$ and $a \vee c$. This however would force $L_{4}^{d} \leqq D$ which is a contradiction and therefore $a \in J(D)$.

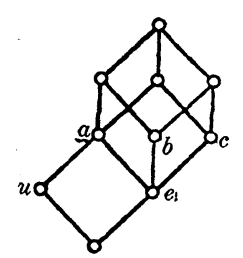

FIgURE (xiv) 
As the partially ordered set $J(D)$ has width 3 , every join-irreducible element of $D$ is either beneath or above at least one of $a, b, c$, so every element of $J(D)$ is comparable with $e$. As $D$ is linearly indecomposable, $e=0$.

It is now clear that by starting with a cover-preserving $C \cong 2^{3}$ inside $D$ and by "going down" until the lowest cube the atoms are elements of $J(D)$ and by also "going up" dually, we end up with a cover-preserving $0-1$-sublattice $E$ isomorphic to $2^{2} \times k$ for some $2 \leqq k \leqq 5$-note that $k \geqq 6$ is impossible, since $L_{1} \leqq 2^{2} \times 6$ ! As $J(E) \leqq$ $J(D)$ by construction and $E$ has the same length as $D$, it follows that $J(E)=J(D)$ and $E=D$.

Proof of Claim 2. As $D$ has breadth two and is linearly indecomposable, there are precisely two atoms say $a$ and $b$. Moreover since $D$ is $\square$-indempocosable, each of these atoms has another upper cover besides $a \vee b$. This produces the interval sublattice of $D$ in Figure (xv).

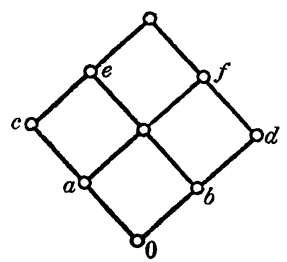

Figure (xv)

Since $L_{2} \not \leq D$, either $e$ or $f$ must be meet irreducible so without loss of generality assume $f \in M(D)$. This implies $D=[c, 1] \cup[0, f]$.

Now suppose $e$ has another upper cover $g \neq e \vee f$ and assume $g \in J(D)$. If $h=\bigvee\{x \in D: x \wedge g=e\}$ we have $e \vee f<h$ since $D$ is $\square$-indecomposable. But $b(D)=2$ implies $[e \vee f, h]$ is a chain and therefore $h \in J(D)$. However this gives $g \wedge h=e=c \vee d \notin J(D)$ which is a contradiction on the projectivity of $D$. Therefore if $e$ has another upper cover $g \neq e \vee f, g$ must be join-reducible. Since any lower cover of $g$ must be in $[c, 1]$ we end up with a join-irreducible $k$ covering $c$ and an interval sublattice of $D$ as in Figure (xvi).

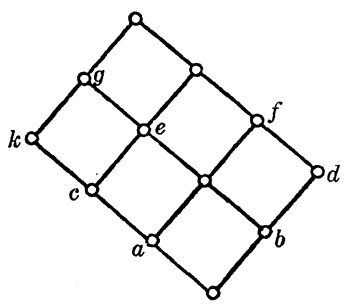

FIGURe (xvi) 
By considering the interval $[a, 1]$ in $D$ and the fact that $f \in M(D)$, it follows that $k$ must have an upper cover in $J(D)$ which produces a sublattice isomorphic to $\mathbf{3} \times \mathbf{5}$. Further extensions cannot happen as $L_{1} \leqq 3 \times 6$.

This finishes the proof of Theorem 5.3.

\subsection{Proof of the main theorem $(\mathscr{G}=\mathscr{C})$ :}

We only have to show that, given any projective $D \in \mathscr{G}$, it follows that $D \in \mathscr{C}$. Let $\left(D_{1}, \cdots, D_{n}\right)$ be the unique $\square$-decomposition of $D$ into $\square$-indecomposables. First observe that in the case $n=1$, we are done, since $2^{4}$ as well as $2^{2} \times 5$ have finite projective covers (see Figure xvii). So we may assume $n>1$; note that all the $D_{j}(1 \leqq j \leqq n)$ are projective. What we show is:

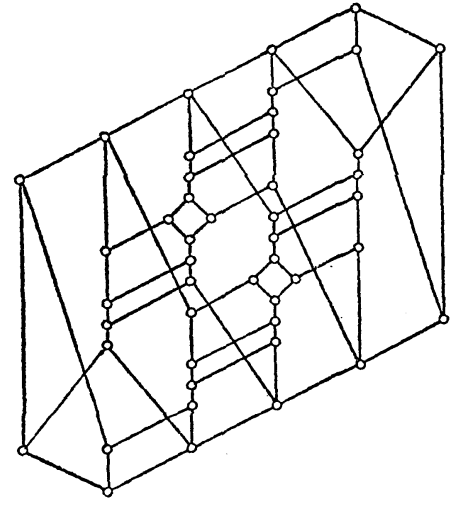

$P_{\mathscr{L}}\left(2^{2} \times 5\right)$

Figure (xviii)

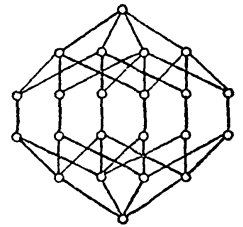

$P_{\mathscr{L}}\left(2^{4}\right)$

(1) that all the intervals of $D$ of the form in Figure (xviii) have finite projective covers, and
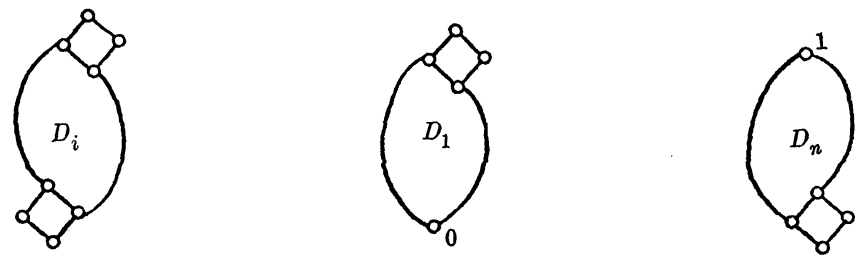

$$
2 \leqq i \leqq n-1
$$

Figure (xviii)

(2) that the projective covers of these parts can be pasted together to give the projective cover of $D$.

To prove (1), it is easy to check that because of 5.3 and $D \in \mathscr{G}$, all the pieces are sublattices of the lattices in Figure (xix). The proof will be concluded by the following two lemmas: 


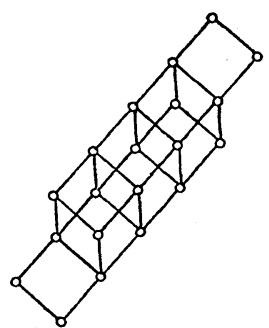

FIgURE (xix)

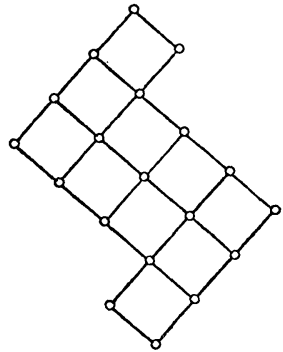

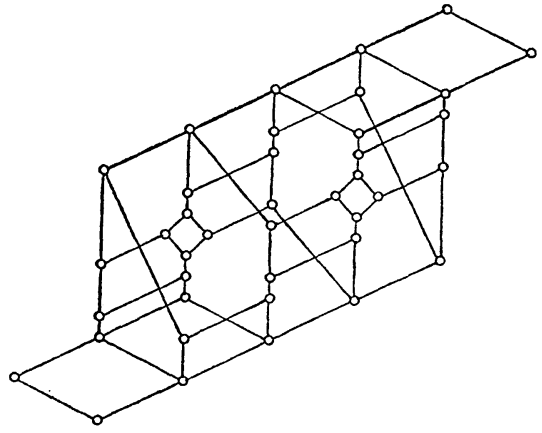

(a)

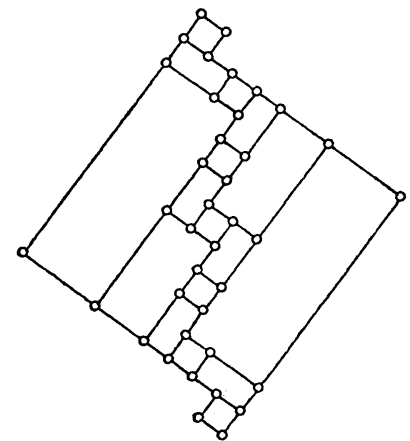

(b)

Figure $(\mathrm{xx})$

5.5. Let $L$ be a finite lattice with finite projective cover $\bar{L}$. If there are two coatoms $a, b$ of $L$ such that $L=[0, a] \cup\{b, 1\}$, then there exist coatoms $\bar{a}, \bar{b}$ in $\bar{L}$ with $\bar{L}=[0, \bar{a}] \cup\{\bar{b}, 1\}$.

Proof. Look at the essential map $f: \bar{L} \rightarrow L$ with lower and upper bound $\alpha, \beta: L \rightarrow \bar{L}$, respectively. Since $f$ is essential, it follows that $\bar{L}=[0, \alpha a] \cup[\beta b, \alpha 1]$. This implies $\alpha 1=\beta 1, \alpha a=\beta a$, and $a b=\beta b$, and by setting $\bar{a}:=\alpha a, \bar{b}:=\beta b$, we get $\bar{L}=[0, \bar{a}] \cup\{\bar{b}, 1\}$.

5.6. Let $L$ be a finite lattice which is $\square$-decomposable into two pieces $[0, a] \cup[b, 1]$ (i.e., $a \wedge b \prec a, b \prec a \vee b$ ). If the sublattices $[0, a \vee b]$ and $[a \wedge b, 1]$ have finite projective covers, then $L$ has a finite projective cover.

Proof. By 5.3 the projective cover $P_{1}$ of $[0, a \vee b]$ has a hook at the top, the cover $P_{2}$ of $[a \wedge b, 1]$ has one at the bottom. Pasting $P_{1}$ and $P_{2}$ together by identifying the hooks gives a lattice $P$ which is easily seen to satisfy $(W)$ and to be the projective cover of $L$.

\section{REFERENCES}

1. K. Baker and A. Hales, Distributive projective lattices, Canad. J. Math., 22 (1970), $472-475$. 
2. R. Balbes, Projective and injective distributive lattices, Pacific J. Math., 21 (1967), 405-420.

3. R. Balbes and A. Horn, Projective distributive lattices, Pacific J. Math., 33 (1970), 273-279.

4. - Projective covers of distributive lattices, Alg. Univ., 6 (1976), 277-290.

5. B. Banaschewski, Projective covers in categories of topological spaces and topological algebras, Proc. of the Topological Conference, Kanpur (1968).

6. G. Birkhoff, Lattice Theory, 3rd edition, Amer. Math. Soc. Colloq. Pub., 25, 1967.

7. B. Davey and B. Sands, An application of Whitman's condition to lattices with no infinite chains, Alg. Univ., 7 (1977), 171-178.

8. A. Day, A simple solution to the word problem for lattices, Canad. Math. Bull., 13 (1970), 253-254.

9. — Splitting lattices generate all lattices, Alg. Univ., 7 (1977), 163-169.

10. Characterizations of finite lattices that are bounded homomorphic images or sublattices of free lattices, Canad. J. Math., 31 (1979), 69-78.

11. R. A. Dean, Sublattices of free lattices, Proc. Symp. in Pure Math. 2, Amer. Math. Soc., (1961), 30-42.

12. R. Freese and J. B. Nation, Projective lattices, Pacific J. Math., 49 (1973), 51-58.

13. F. Galvin and B. Jónsson, Distributive sublattices of a free lattice, Canad. J. Math., 13 (1961), 265-272.

14. H. Gaskill, Sublattices of free lattices generated from distributive lattices, preprint (1975).

15. - Distributive lattices which generate finite sublattices of free lattices, preprint (1976).

16. H. Gaskill, G. Grätzer and C. R. Platt, Sharp transferable lattices, Canad. J. Math., 27 (1975), 1246-1262.

17. H. Gaskill and C. R. Platt, Sharp transferability and finite sublattices of free lattices, Canad. J. Math., 27 (1975), 1036-1041.

18. B. Jónsson, Sublattices of a free lattice, Canad. J. Math., 13 (1961), 256-264.

19. B. Jónsson and J. Kiefer, Finite sublattices of a free lattice, Canad. J. Math., 14 (1962), 487-497.

20. B. Jónsson and J. B. Nation, A report on finite sublattices of a free lattice, Collog. Math. Soc. Janos Bolyai 17. Contributions to Universal Algebra, (Szeged), 1975, 223-257. 21. A. Kostinsky, Projective lattices and bounded homomorphisms, Pacific J. Math., 40 (1972), 111-119.

22. R. McKenzie, Equational bases and non-modular lattice varieties, Trans. Amer. Math. Soc., 174 (1972), 1-43.

23. P. Whitman, Free lattices I, Ann. of Math., 42 (1941), 325-330.

24. - Free lattices II, Ann. of Math., 43 (1942), 104-115.

Received May 31, 1978.

LAKEHEAD UNIVERSITY

Thunder Bay, Ontario, Canada P7B 5E1

MEMORIAL UNIVERSITY

ST JoHns, NewfoundLand

Canada A1B 3X7

AND

FB4 AG1

TeChNische Hochschule

6100 DARMSTADT, BRD 



\section{PACIFIC JOURNAL OF MATHEMATICS}

EDITORS

DONALD BABBITT (Managing Editor)

University of California

Los Angeles, California 90024

HUGo RossI

University of Utah

Salt Lake City, UT 84112

C. C. MOORE

J. DUGUNDJI

Department of Mathematics

University of Southern California

Los Angeles, California 90007

R. FinN AND J. MiLgraM

Stanford University

Stanford, California 94305

University of California

Berkeley, CA 94720

\section{ASSOCIATE EDITORS}

E. F. BrCKENBACH

B. H. NeUmanN

F. WOLF

K. YoshidA

\section{SUPPORTING INSTITUTIONS}

UNIVERSITY OF BRITISH COLUMBIA

UNIVERSITY OF SOUTHERN CALIFORNIA

CALIFORNIA INSTITUTE OF TECHNOLOGY

STANFORD UNIVERSITY

UNIVERSITY OF CALIFORNIA

UNIVERSITY OF HAWAII

MONTANA STATE UNIVERSITY

UNIVERSITY OF TOKYO

UNIVERSITY OF NEVADA, RENO

UNIVERSITY OF UTAH

NEW MEXICO STATE UNIVERSITY

WASHINGTON STATE UNIVERSITY

OREGON STATE UNIVERSITY

UNIVERSITY OF WASHINGTON

UNIVERSITY OF OREGON 


\section{Pacific Journal of Mathematics}

\section{Vol. 81, No. $1 \quad$ November, 1979}

Thomas E. Armstrong, Simplicial subdivision of infinite-dimensional compact cubes ..................................... 1

Herbert Stanley Bear, Jr., Approximate identities and pointwise convergence ................................

Richard David Bourgin, Partial orderings for integral representations on convex sets with the Radon-Nikodým property..................

Alan Day, Herbert S. Gaskill and Werner Poguntke, Distributive lattices

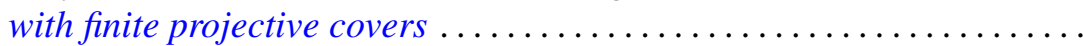

Heneri Amos Murima Dzinotyiweyi and Gerard L. G. Sleijpen, A note on measures on foundation semigroups with weakly compact orbits ......

Ronald James Evans, Resolution of sign ambiguities in Jacobi and Jacobsthal sums ...................................

John Albert Fridy, Tauberian theorems via block dominated matrices ......

Matthew Gould and Helen H. James, Automorphism groups retracting onto

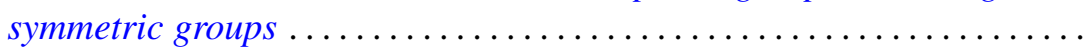

Kurt Kreith, Nonlinear differential equations with monotone solutions . . . . 101

Brian William McEnnis, Shifts on indefinite inner product spaces........ 113

Joseph B. Miles, On entire functions of infinite order with radially

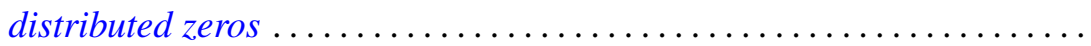

Janet E. Mills, The idempotents of a class of 0-simple inverse semigroups ...............................

Edward Jean Moulis, Jr., Generalizations of the Robertson functions ...

Richard A. Moynihan and Berthold Schweizer, Betweenness relations in probabilistic metric spaces.......................

Stanley Ocken, Perturbing embeddings in codimension two ....

Masilamani Sambandham, On the average number of real zeros of a class of random algebraic curves.

Jerry Searcy and B. Andreas Troesch, A cyclic inequality and a related eigenvalue problem.

Roger R. Smith and Joseph Dinneen Ward, $M$-ideals in $B\left(l_{p}\right)$...

Michel Talagrand, Deux généralisations d'un théorème de I. Namioka ..

Jürgen Voigt, $O n Y$-closed subspaces of $X$, for Banach spaces $X \subset Y$;

existence of alternating elements in subspaces of $C(J)$

Sidney Martin Webster, On mapping an $n$-ball into an $(n+1)$-ball in complex spaces

David J. Winter, Triangulable subalgebras of Lie p-algebras ... 